\title{
Glucagon-like peptide-1 receptor stimulation increases blood pressure and heart rate and activates autonomic regulatory neurons
}

\author{
Hiroshi Yamamoto, ${ }^{1}$ Charlotte E. Lee, ${ }^{1}$ Jacob N. Marcus, ${ }^{1}$ Todd D. Williams, ${ }^{2}$ \\ J. Michael Overton, ${ }^{2}$ Marisol E. Lopez, ${ }^{1}$ Anthony N. Hollenberg, ${ }^{1}$ Laurie Baggio, ${ }^{3}$ \\ Clifford B. Saper, ${ }^{4}$ Daniel J. Drucker, ${ }^{3}$ and Joel K. Elmquist ${ }^{1,4}$
}
${ }^{1}$ Department of Medicine and Division of Endocrinology, Beth Israel Deaconess Medical Center, Harvard Medical School, Boston, Massachusetts, USA
${ }^{2}$ Department of Nutrition, Food, and Exercise Science, Program in Neuroscience, Florida State University, Tallahassee, Florida, USA
${ }^{3}$ Department of Medicine, Toronto General Hospital, and the Banting and Best Diabetes Centre, University of Toronto, Toronto, Ontario, Canada
${ }^{4}$ Department of Neurology, Beth Israel Deaconess Medical Center, and Program in Neuroscience, Harvard Medical School, Boston, Massachusetts, USA

\begin{abstract}
Glucagon-like peptide-1 (GLP-1) released from the gut functions as an incretin that stimulates insulin secretion. GLP-1 is also a brain neuropeptide that controls feeding and drinking behavior and gastric emptying and elicits neuroendocrine responses including development of conditioned taste aversion. Although GLP-1 receptor (GLP-1R) agonists are under development for the treatment of diabetes, GLP-1 administration may increase blood pressure and heart rate in vivo. We report here that centrally and peripherally administered GLP-1R agonists dose-dependently increased blood pressure and heart rate. GLP-1R activation induced c-fos expression in the adrenal medulla and neurons in autonomic control sites in the rat brain, including medullary catecholamine neurons providing input to sympathetic preganglionic neurons. Furthermore, GLP-1R agonists rapidly activated tyrosine hydroxylase transcription in brainstem catecholamine neurons. These findings suggest that the central GLP-1 system represents a regulator of sympathetic outflow leading to downstream activation of cardiovascular responses in vivo.
\end{abstract}

J. Clin. Invest. 110:43-52 (2002). doi:10.1172/JCI200215595.

\section{Introduction}

Glucagon-like peptide-1 (GLP-1) acts as a nutrientstimulated incretin secreted from enteroendocrine $\mathrm{L}$

Received for publication April 3, 2002, and accepted in revised form May 28, 2002.

Address correspondence to: Joel K. Elmquist, Division of Endocrinology, Beth Israel Deaconess Medical Center, 347 Research North, 99 Brookline Avenue, Boston, Massachusetts 02215, USA. Phone: (617) 667-3218; Fax: (617) 667-2927; E-mail: jelmquis@caregroup.harvard.edu. Conflict of interest: D.J. Drucker is a consultant to Amylin Pharmaceuticals Inc. and Conjuchem Inc.

Nonstandard abbreviations used: glucagon-like peptide- 1 (GLP-1); GLP-1 receptor (GLP-1R); dipeptidyl peptidase-4 (DPP-IV); exendin-4 (EXN-4); nucleus of the solitary tract (NTS); intracerebroventricular(ly) (i.c.v.); intravenous(ly) (i.v.); immunoreactivity (IR); Fos-like immunoreactivity (Fos-IR); paraventricular hypothalamus (PVH); corticotropin-releasing hormone $(\mathrm{CRH})$; blood pressure (BP); heart rate (HR); Fluorogold (FG); intermediolateral cell column (IML); mean arterial blood pressure (MAP); pyrogen-free saline (PFS); tyrosine hydroxylase (TH); protected least significant difference (PLSD); heteronuclearRNA (hnRNA); avidin-biotin complex $(\mathrm{ABC})$; diaminobenzidine tetrahydrochloride (DAB); lateral hypothalamic area (LHA); external lateral parabrachial nucleus (PBel); locus coeruleus (LC); A5 catecholamine cell group (A5); area postrema (AP); rostral ventrolateral medulla (RVLM); causal ventrolateral medulla (CVLM);

hypothalamo-pituitary-adrenocortical (HPA). cells in the intestine (1). GLP-1 promotes nutrient assimilation via stimulation of glucose-dependent insulin release and inhibition of glucagon secretion. Native GLP-1 is rapidly degraded by dipeptidyl peptidase-IV (DPP-IV) in the bloodstream. In contrast, modified peptidase-resistant GLP-1 analogues and lizard exendin-4 (EXN-4) are highly resistant to degradation by DPP-IV, exhibit longer half-lives, and are substantially more potent than native GLP- $1(2,3)$. Accordingly, GLP-1 analogues and EXN-4 are currently being assessed in clinical trials for the treatment of subjects with type 2 diabetes $(4,5)$.

GLP-1 is also a neuropeptide synthesized by neurons in the caudal regions of the nucleus of the solitary tract (NTS) (6). In contrast to the limited sites of GLP-1 synthesis in the CNS, GLP-1-immunoreactive fibers and GLP-1 receptors (GLP-1Rs) are quite widespread throughout the brain (6-8). Not surprisingly, GLP-1 exerts diverse actions in the CNS, including inhibition of food and water intake. Intracerebroventricular (i.c.v.) administration of GLP-1 induces Fos-like immunoreactivity (Fos-IR) in the paraventricular nucleus of the hypothalamus $(\mathrm{PVH})(9,10)$. Peripheral administration of EXN-4 reduces fasting and postprandial glucose and energy intake in humans (11). Whether GLP-1 acts specifically on inhibitory feeding centers to produce 
satiety or acts as a mediator of aversive stimuli remains uncertain, as several experiments demonstrate development of taste aversion $(12,13)$ and activation of signaling pathways mediating interoceptive stress responses (14-16) following GLP-1 administration. Consistent with the involvement of GLP-1 signaling in stress responses, central administration of GLP-1 activates neurons containing corticotropin-releasing hormone (CRH) in the PVH and elevates plasma corticosterone (17). Furthermore, both GLP-1 and EXN-4 produce chronotropic and pressor responses in anesthetized animals (18-21); however, whether these effects are direct, due to activation of cardiac GLP-1Rs, or indirect remains unclear. Despite the accumulating data linking GLP-1R signaling to autonomic and neuroendocrine responses, the neural pathways underlying these actions of central GLP-1 are not fully understood. To determine the mechanisms and potential physiological significance of central GLP-1 pathways impinging on sympathetic outflow systems, we investigated the distribution of GLP-1-activated neurons that innervate sympathetic preganglionic neurons and assessed GLP-1 effects on cardiovascular parameters.

\section{Methods}

Animals and surgical procedures. Adult male pathogen-free Sprague-Dawley rats (250-350 g; Taconic, Germantown, New York, USA) were housed in a light-controlled (12 hours on, 12 hours off) and temperature-controlled $\left(21.5-22.5^{\circ} \mathrm{C}\right)$ environment. The animals and procedures used were approved by the Harvard Medical School and Beth Israel Deaconess Medical Center institutional animal care and use committees.

Rats were anesthetized with isoflurane (induction 5\%, maintenance $2 \%$ ) and were implanted in the abdominal aorta with a catheter coupled with a sensor and transmitter (TA11PA-C40; Data Sciences International, St. Paul, Minnesota, USA) (22). Telemetry signals were received by an antenna below the rat's cage that relays the signals to a signal processor (DataQuest A.R.T. Gold version 2.1 system; Data Sciences International) connected to a Compaq Deskpro PC (Compaq Computer Corp., Houston, Texas). These transmitters allow remote monitoring of blood pressure (BP) and heart rate $(\mathrm{HR})$ in unanesthetized and unrestrained animals. In the same surgical session, rats were implanted intravenously (i.v.) with a silastic catheter into the femoral vein (23) or i.c.v. with a guide cannula into the lateral ventricle (24). In a subset of the animals, the retrograde tracer Fluorogold (FG) $(2.5 \%$ in pyrogen-free saline) (Fluorochrome, Denver, Colorado, USA) was injected with a glass micropipette and air pressure injection system $(25,26)$. The injections included the intermediolateral cell column (IML) and consisted of two injections of $50 \mathrm{nl}$ in the rostral (T1-T4) or caudal (T6-T10) thoracic spinal cord.

Ten to fourteen days after surgery, base-line mean arterial blood pressure (MAP) and HR were recorded for the preceding day and night, and rats were injected with
EXN-4 (30, 300, or 3000 ng per rat i.v. or 3, 30, or 300 ng per rat i.c.v.; $n=4-6$ for each group) or pyrogen-free saline (PFS) $(0.25 \mathrm{ml}$ i.v. or $5 \mu$ li.c.v., total volume per rat; $n=4-6$ for each group). In our initial experiments, we observed that both i.v. and i.c.v. injections of PFS induced a transient increase in MAP and HR. Thus, we used a long line to avoid a handling stress during injection in a subsequent experiment. The long line was connected 2 hours before i.c.v. injection of GLP-1(7-36) (300 ng per rat; $n=3)$ or PFS ( $5 \mu$ l total volume per rat; $n=3)$. The action of [des-His ${ }_{1}$, Glu $\left.{ }_{9}\right]$ exendin-4 of the GLP-1R antagonist (13) was investigated by i.c.v. administration of $1 \mu \mathrm{g}$ of the peptide 30 minutes before i.c.v. administration of $30 \mathrm{ng}$ of $\mathrm{EXN}-4$. All drugs were given to unrestrained rats between 2 and 5 hours after lights on. The cardiovascular data were collected and stored in 1-minute bins, and, before further analysis, the data were averaged into 10 -minute bins. Results are expressed as means \pm SEM from groups of four to six rats. Statistical comparisons between animals were performed at each time. ANOVA for repeated measurement was used to estimate dose-dependent effects within each group, and the differences between groups in each time were assessed by Fisher's protected least significant difference (PLSD).

Animal perfusion and histology. Two hours after administration of peptides or PFS, rats were deeply anesthetized with isoflurane and perfused transcardially with diethyl pyrocarbonate-treated $0.9 \%$ saline followed by $10 \%$ neutral formalin (Sigma-Aldrich, St. Louis, Missouri, USA). For tyrosine hydroxylase (TH) heteronuclearRNA (hnRNA) in situ hybridization studies, rats were perfused 0,15 , or 30 minutes or $1,2,6$, or 24 hours after administration of EXN-4 or PFS $(n=3$ for each group). Before perfusion, blood was collected from the heart, and blood glucose was measured using a glucose oxidase-based method (LifeScan OneTouch; Johnson \& Johnson, Milpitas, California, USA). Tissues were removed and postfixed in the same fixative for 4 hours, submerged in $20 \%$ sucrose, and cut at $30 \mu \mathrm{m}$ ( $1: 5$ series).

Immunobistochemistry. The procedures for immunohistochemistry were performed as reported previously $(23,26,27)$. For single-label immunohistochemistry, sections were pretreated with $0.3 \%$ hydrogen peroxide in PBS for 30 minutes, and then in 3\% normal donkey serum (Jackson ImmunoResearch Laboratories Inc., West Grove, Pennsylvania, USA) with $0.25 \%$ Triton $\mathrm{X}-100$ in PBS (PDT), followed by overnight incubation in Fos rabbit primary antisera (Ab5; Oncogene Research Products, Cambridge, Massachusetts, USA; 1:100,000 dilution in PDT) at room temperature. After washing in PBS, sections were incubated in biotinylated donkey anti-rabbit IgG (Jackson ImmunoResearch Laboratories Inc.; 1:1,000) and then incubated with avidin-biotin complex (Vector Elite ABC Kit; Vector Laboratories Inc., Burlingame, California, USA; 1:500 in PBS). A combination of $0.04 \%$ diaminobenzidine tetrahydrochloride (DAB; Sigma-Aldrich), 0.01\% nickel ammonium sulfate (Fisher Scientific Co., Pittsburgh, 
Pennsylvania, USA), $0.01 \%$ cobalt chloride (Fisher Scientific Co.), and $0.01 \%$ hydrogen peroxide dissolved in PBS was used for the chromogen reaction with two successive rinses in PBS. The tissue sections were mounted onto subbed slides, air-dried, dehydrated in alcohol, cleared in xylene, and then coverslipped with Permaslip (Alban Scientific, St. Louis, Missouri, USA).

For double-label immunohistochemistry, the sections stained with Fos were blocked and then incubated overnight in FG rabbit primary antisera (Chemicon International, Temecula, California, USA; 1:20,000 in PDT) or mouse anti-TH (Chemicon International; 1:20,000 in PDT). The sections were processed as described above using DAB as a chromogen.

For triple-label immunohistochemistry, the tissue sections were incubated in Fos antisera and were processed as described above but incubated in the DAB solution without nickel sulfate and cobalt chloride, resulting in a brown nuclear reaction product. Sections were then incubated in FG rabbit primary antisera and mouse anti$\mathrm{TH}$ overnight at room temperature. After washing in PBS, sections were incubated in FITC-conjugated donkey anti-rabbit IgG (Jackson ImmunoResearch Laboratories Inc.; 1:200 in PDT) and Cy3-conjugated donkey anti-mouse IgG (Jackson ImmunoResearch Laboratories Inc.; 1:200 in PDT). The Fos-IR was observed with brightfield optics, the TH-immunoreactivity (TH-IR) with a rhodamine filter system, and the retrograde label with a FITC filter system.

In situ bybridization histochemistry. A fragment of the rat TH gene (28) including intron 2 was amplified by PCR with rat genomic DNA (BD Biosciences Clontech, Palo Alto, California, USA) as a template. The following primers were used for amplification: primer $1,5^{\prime}$ TGTCTCGGGCTGTAAAAGTATTTGA-3'; and primer 2, 5'-
AgtGgtgaAtTTtGgCTTCAAATGT-3'. The insert was cloned into the pGEM-T Easy Vector (Promega Corp., Madison, Wisconsin, USA). The PCR product was cloned and the identity of the insert as TH intron was confirmed by sequence analysis and comparison to the GenBank database (accession number AF014956). For generation of $550 \mathrm{bp}$ of antisense ${ }^{35} \mathrm{~S}$-labeled cRNA, the plasmid was linearized by digestion with $\mathrm{NcoI}$ and subjected to in vitro transcription with SP6 polymerase. The protocol for in situ hybridization histochemistry was a modification of that previously reported by our laboratory (29) and several other laboratories (30). Control procedures to confirm the specificity of our in situ hybridization histochemistry procedures included hybridization with antisense probes after tissue pretreatment with RNase A $(200 \mu \mathrm{g} / \mathrm{ml})$.

Estimates of cell counts. In all procedures, the estimates of cell counts (Fos, TH, and FG) were done with a grid reticule and a $10 \times$ objective by an observer blinded to the treatment groups $(23,26)$. The data were not corrected for double counting and a stereological technique was not used, because the objects we were counting (nuclei and retrogradely labeled cells) did not change in size, and section thickness did not vary between groups. Hence, as all double-label studies are inherently qualitative, our results are meant to provide relative data but are not meant to be accurate estimates of absolute cell counts. Data were analyzed by ANOVA and differences between groups by Fischer's PLSD.

Production of photomicrographs. Photomicrographs were captured with a digital camera (Spot3.0; Diagnostic Instruments, Sterling Heights, Michigan, USA) mounted directly on the microscope (Axioskop 2; Carl Zeiss Inc., Thornwood, New York, USA) and an Apple Macintosh G3 (Apple Computer Inc., Cupertino, California, USA) computer. Image editing software (Adobe Photoshop; Adobe Systems Inc., Mountain View, California, USA) was used to combine photomicrographs into plates. Only the sharpness, contrast, and brightness were adjusted. All figures were printed on a dye-sublimation printer (Kodak 8670; Eastman Kodak Co. Scientific Imaging Systems, New Haven, Connecticut, USA). For drawings (see Figure 4), cytoarchitectonic details were added using a camera lucida.

\section{Results}

GLP-1 agonists increase BP and HR. To ascertain the effects of GLP-1R activation on cardiovascular responses in non-anes-

\footnotetext{
Figure 1

Effects of i.c.v. or i.v. administration of EXN-4 on MAP and HR. Both i.v. EXN-4 (a and b) and i.c.v. EXN-4 ( $\mathbf{c}$ and $\mathbf{d}$ ) dose-dependently increase MAP (a and $\mathbf{c}$ ) and HR (b and $\mathbf{d})$. EXN-4 was injected at " 0 ." ${ }^{*} P<0.05,{ }^{*} P<0.01$.
} 


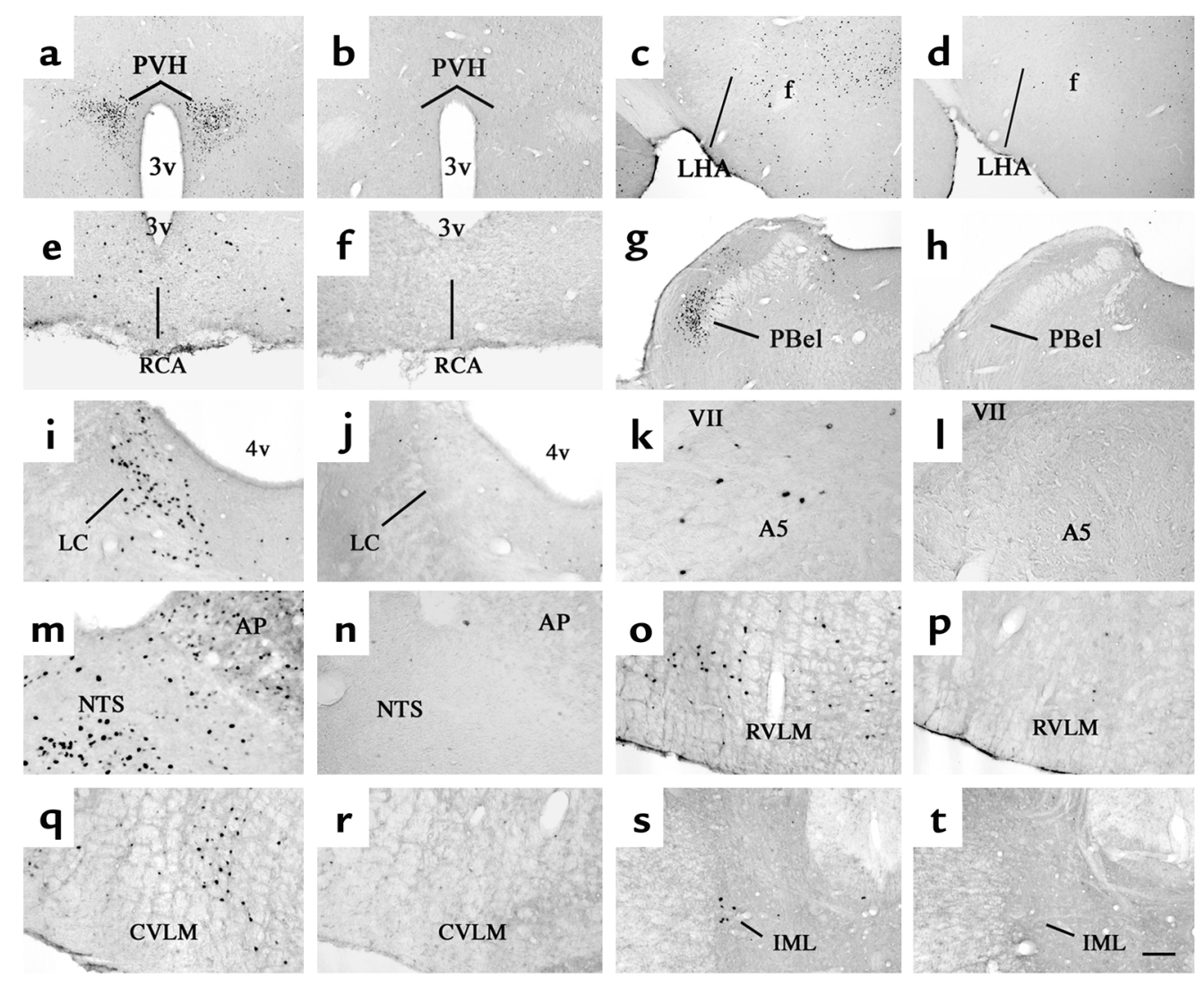

\section{Figure 2}

Distribution of i.v. EXN-4-induced Fos-IR in the brain. A series of photomicrographs demonstrates Fos-IR in neurons 2 hours after i.v. administration of EXN-4 (first and third columns) or PFS (second and fourth columns) in several brain regions. These regions include (a and $\mathbf{b}$ ) the paraventricular nucleus of the hypothalamus (PVH); (c and d) the lateral hypothalamic area (LHA); (e and $\mathbf{f})$ the arcuate nucleus (Arc) and the retrochiasmatic area (RCA); $(\mathbf{g}$ and $\mathbf{h})$ the external lateral subdivision of the parabrachial nucleus (PBel); ( $\mathbf{i}$ and $\mathbf{j})$ the locus coeruleus (LC); ( $\mathbf{k}$ and $\mathbf{I})$ the A5 cell group (A5); ( $\mathbf{m}$ and $\mathbf{n}$ ) the area postrema (AP) and the NTS; (o and $\mathbf{p})$ the rostral ventrolateral medulla (RVML); ( $\mathbf{q}$ and $\mathbf{r}$ ) the caudal ventrolateral medulla (CVLM); and (s and $\mathbf{t})$ the IML in the spinal cord. $3 \mathrm{v}$, third ventricle; $f$, fornix; $4 \mathrm{v}$, fourth ventricle; VII, facial nerve. Scale bar $=250 \mu \mathrm{m}$ in $\mathbf{a}-\mathbf{d}, \mathbf{g}$, and $\mathbf{h}$, and $100 \mu \mathrm{m}$ in $\mathbf{e}, \mathbf{f}$, and $\mathbf{i}-\mathbf{t}$.

thetized rats, we assessed BP and HR in freely moving rats using telemetric monitoring. MAP and HR transiently increased after handling and after i.v. or i.c.v. administration of PFS. Both parameters returned to basal levels within 40-50 minutes (Figure 1, a-d). In contrast, i.v. administration of EXN-4 produced a dosedependent increase in MAP and HR (Figure 1, a and b). Similarly, i.c.v. administration of EXN-4 dose-dependently increased MAP and HR (Figure 1, $c$ and d). MAP and HR remained significantly increased for up to 2 hours after both i.v. (300 and $3000 \mathrm{ng}$ ) and i.c.v. (30 and $300 \mathrm{ng}$ ) administration of EXN-4.

As EXN-4 is a protease-resistant long-acting lizard GLP$1 \mathrm{R}$ agonist, we also examined MAP and HR after i.c.v. administration of native mammalian GLP-1(7-36 amide). Intracerebroventricular GLP-1 (300 ng) also produced a robust increase in MAP and HR that remained significantly increased up to 60 minutes after administration. The MAP and HR responses were shorter in duration than that induced by EXN-4 and returned to base line by 60 minutes. The specificity of the responses for the GLP$1 \mathrm{R}$ was confirmed by the demonstration that pretreat- ment with i.c.v. administration of $1 \mu \mathrm{g}$ of [des$\mathrm{His}_{1}, \mathrm{Glu}_{9}$ ] exendin-4 (13) completely blocked the effects of $30 \mathrm{ng}$ of i.c.v. EXN-4 on MAP and HR.

As peripheral GLP-1 increases insulin secretion in a glucose-dependent fashion, we assessed whether the observed cardiovascular responses were secondary to GLP-1 agonist-induced hypoglycemia. Blood glucose levels increased modestly after i.c.v. administrations of both EXN-4 (basal, $96 \pm 1.6$; 15 minutes, $105 \pm 3.9 ; 30$ minutes, $109 \pm 2.4 ; 60$ minutes, $110 \pm 3.0 ; 120$ minutes, $110 \pm 1.4$; mean \pm SEM) and PFS (basal, $98 \pm 2.0 ; 15$ minutes, $106 \pm 4.9$; 30 minutes, $104 \pm 2.4$; 60 minutes, $108 \pm 5.6$; 120 minutes, $106 \pm 4.3$; mean \pm SEM). Importantly, i.c.v. administration of EXN-4 did not lower blood glucose at any time points after the injection.

Distribution of EXN-4-induced Fos-IR. To determine potential downstream mediators of GLP-1R activation leading to an increase in MAP and HR, we investigated the distribution of Fos-IR 2 hours after i.v. or i.c.v. EXN4 administration at doses that increase MAP and HR (i.v., 300 ng; i.c.v., 30 ng). Intravenous administration induced Fos-IR in several autonomic control sites (31) 
that showed little or no Fos-IR after PFS injection (Figure 2, a-t). These regions included the $\mathrm{PVH}$, the lateral hypothalamic area (LHA), the arcuate nucleus (Arc) and the retrochiasmatic area (RCA), the external lateral parabrachial nucleus (PBel), the locus coeruleus (LC), the A5 catecholamine cell group (A5), the area postrema (AP), the NTS, the rostral ventrolateral medulla (RVLM), the caudal ventrolateral medulla (CVLM), and the IML of the spinal cord. The distribution of Fos-immunoreactive neurons induced by i.c.v. EXN-4 was similar to that induced by i.v. EXN-4 (Figure 3, a-t).

Distribution of EXN-4-activated neurons projecting to the spinal cord. To ascertain whether the observed increase in MAP and HR was due to activation of GLP-1-sensitive cell groups that also innervate sympathetic preganglionic neurons, we injected the retrograde tracer FG) into the spinal thoracic cord centered on the IML $(26,27)$, with or without concomitant i.c.v. EXN-4 administration. The distribution of retrogradely labeled (FG-immunoreactive) neurons in the hypothalamus and the brainstem was similar to that in previous reports $(26,27,32,33)$. Following administration of EXN-4, we observed many double-labeled cells (Fos + FG) in the hindbrain, including the LC, the A5, and the RVLM (see Figure 5, n and p; Table 1). In the NTS, despite the existence of separate groups of neurons expressing either Fos-IR or FG-IR, we did not observe any double-labeled neurons. Many doublelabeled neurons were observed in the hypothalamus, including the PVH, the Arc/RCA, and the LHA (Figure 4; Figure 5, a-c; Table 1).

GLP-1R agonists activate catecholamine neurons. As the topographical distribution of neurons displaying EXN4-induced Fos-IR in the brainstem resembles the distribution of catecholamine neurons that are known to play essential roles in regulating the sympathetic nervous system (34-36), we assessed whether medullary catecholamine neurons were activated by doses of EXN-4 that increase MAP and HR. We found double-labeled (TH + Fos) neurons following i.c.v. EXN-4 administration in the LC, the A5, the AP, the NTS, the RVLM, and the CVLM (Figure 5, $\mathrm{g}-\mathrm{i}, \mathrm{m}$, and o).

Using triple labeling, we observed that approximately $25 \%$ of TH-immunoreactive neurons in the RVLM contain Fos-IR following i.c.v. EXN-4 and also project to the IML (Figure 5, $\mathrm{m}$ and $\mathrm{n}$ ). Similarly, approximately $30 \%$ of the TH-immunoreactive neurons in the A5 group contained both Fos and FG (Figure 5, o and

\section{Figure 3}

Distribution of i.c.v. EXN-4-induced Fos-IR in the brain and the adrenal gland. A series of photomicrographs demonstrates Fos-IR in neurons 2 hours after i.c.v. administration of EXN-4 (a-t, first and third columns; $\mathbf{v}-\mathbf{x})$ or PFS (a-t, second and fourth columns; $\mathbf{u}$ ) in several brain regions and the adrenal gland. The brain regions include ( $\mathbf{a}$ and $\mathbf{b}$ ) the $\mathrm{PVH}$; (c and d) the LHA; (e and f) the Arc/RCA; ( $\mathbf{g}$ and $\mathbf{h}$ ) the PBel; ( $\mathbf{i}$ and j) the LC; (k and I) the A5; ( $\mathbf{m}$ and $\mathbf{n}$ ) the AP and the NTS; ( $\mathbf{o}$ and $\mathbf{p}$ ) the RVML; ( $q$ and $\mathbf{r}$ ) the CVLM; and (s and $\mathbf{t}$ ) the IML in the spinal cord. In the adrenal gland, after administration of PFS, little Fos-IR is visible (u). After i.c.v. EXN-4 at subthreshold dose $(\mathbf{v} ; 3 \mu \mathrm{g})$, a little Fos-IR is visible. In contrast, i.c.v. EXN-4 at threshold dose $(\mathbf{w} ; 30 \mu \mathrm{g})$ or a higher dose $(\mathbf{x} ; 300 \mu \mathrm{g})$ induces Fos-IR in the adrenal medulla. Note that at the higher dose (x), Fos-IR is also visible in the cortex. Scale bar $=500 \mu \mathrm{m}$ in $\mathbf{a - d}, \mathbf{g}$, and $\mathbf{h} ; 200 \mu \mathrm{m}$ in $\mathbf{e}, \mathbf{f}$, and $\mathbf{i}-\mathbf{t}$; and $50 \mu \mathrm{m}$ in $\mathbf{u}-\mathbf{x}$.
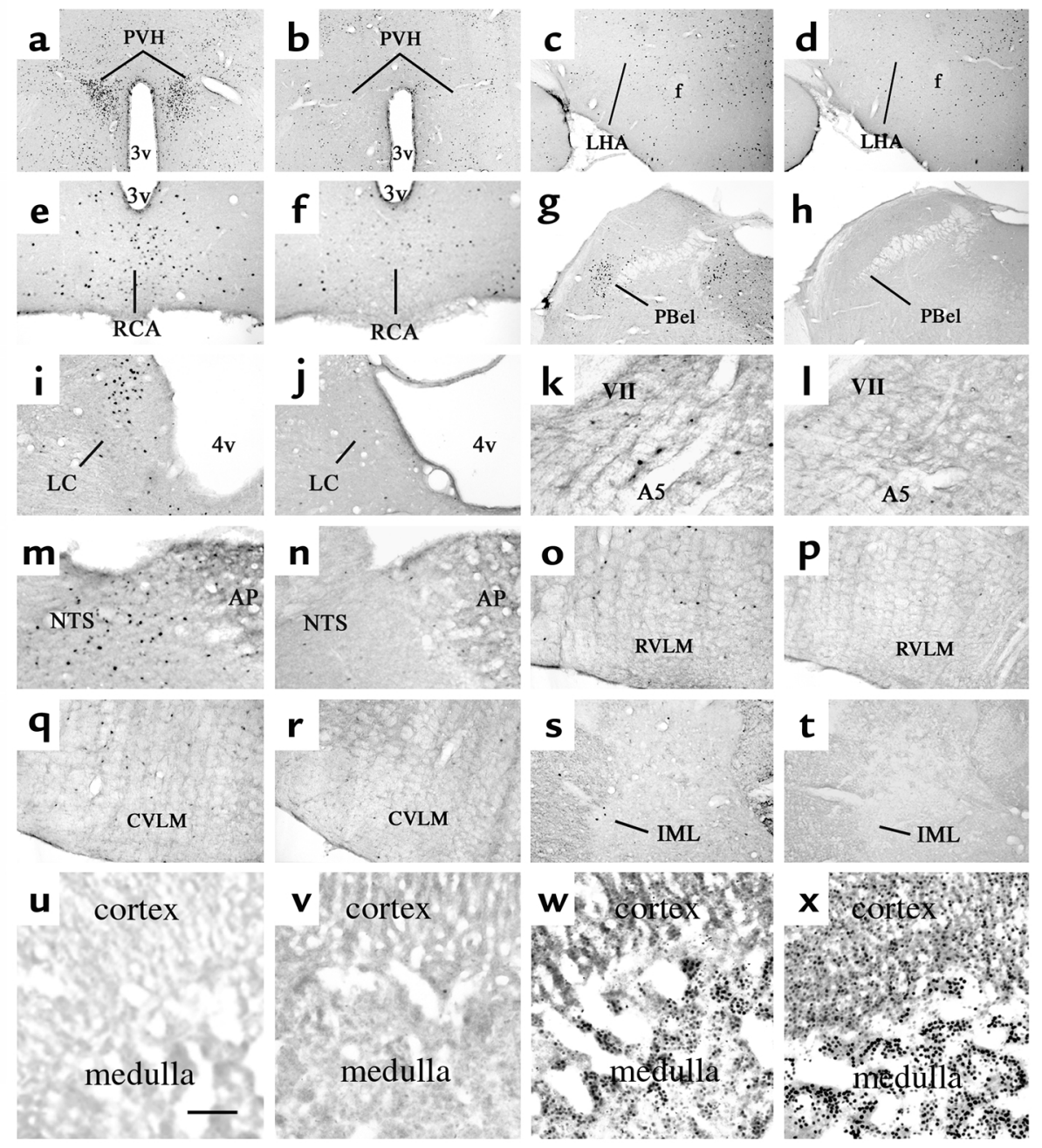

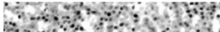
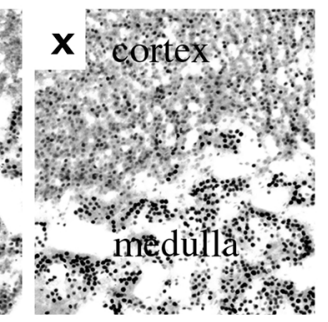

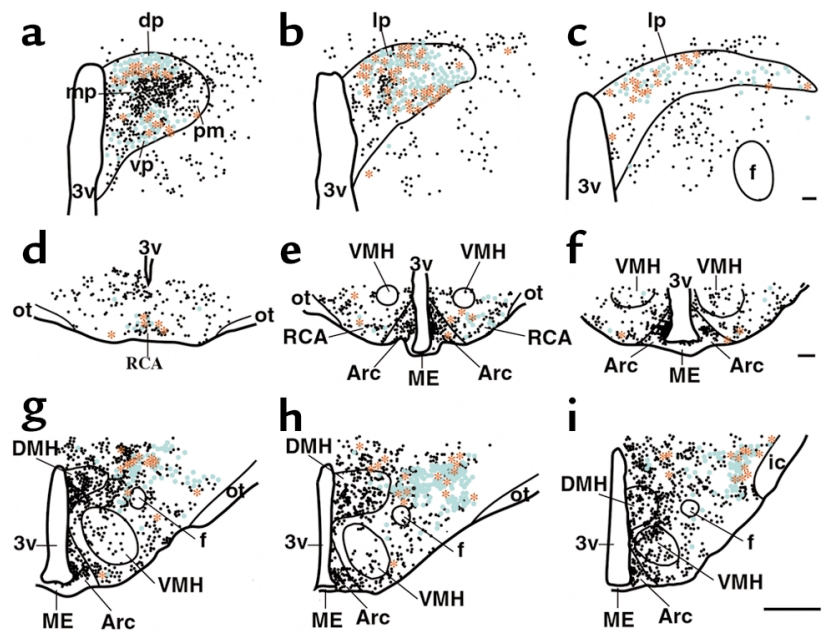

\section{Figure 4}

EXN-4-activated neurons projecting to the spinal cord. A series of line drawings demonstrates the distribution of Fos-IR (black circles), FG-IR (blue circles), and double-labeled (red stars) neurons in three rostral-to-caudal levels of the PVH $(\mathbf{a}-\mathbf{c})$, the $\operatorname{Arc} / \mathrm{RCA}(\mathbf{d}-\mathbf{f})$, and the LHA ( $\mathbf{g}-\mathbf{i})$ of the hypothalamus after i.c.v. injection of EXN-4, in animals with FG injection into the spinal cord. Scale bar $=250 \mu \mathrm{m}$ in $\mathbf{a}-\mathbf{c}$ and $1,000 \mu \mathrm{m}$ in $\mathbf{d}-\mathbf{i}$. Divisions of the PVH: dp, dorsal parvocellular; mp, medial parvocellular; pm, posterior magnocellular; vp, ventral parvocellular; Ip, lateral parvicellular; ic, internal capsule. $\mathrm{VMH}$, ventromedial nucleus of the hypothalamus; $\mathrm{DMH}$, dorsomedial nucleus of the hypothalamus; ME, median eminence.

p). In contrast, although we observed many doublelabeled neurons (Fos $+\mathrm{TH}$ ) in the NTS, we did not observe any triple-labeled neurons in this region. In the LC, nearly all retrogradely labeled neurons activated by EXN-4 contained TH.

Previous studies have demonstrated that stimuli that induce sympathoadrenal response also display Fos-IR in the adrenal gland (37). To determine whether a dose of i.c.v. EXN-4 that increases MAP and HR is associated with activation of both central and peripheral catecholaminergic pathways, we investigated the distribution of Fos-IR in the adrenal gland 2 hours after EXN-4 administration. Intracerebroventricular administration of EXN-4 at doses that increase MAP and HR also markedly induced Fos-IR in the adrenal medulla (Figure 3, w and $\mathrm{x}$ ). PFS injection or EXN-4 injection at subthreshold doses ( $3 \mathrm{ng}$ ) induced little or no Fos (Figure 3 , $u$ and v). The higher dose of i.c.v. EXN-4 (300 ng) also induced Fos-IR in the adrenal cortex (Figure 3x).

GLP-1R agonists activate TH gene transcription. TH catalyzes the rate-limiting step in catecholamine biosynthesis, and the concentration and activity of $\mathrm{TH}$ correlate with neuronal activity (38). Both pharmacological stimulation and electrical stimulation increase TH transcription $(36,39)$. However, basal expression of $\mathrm{TH}$ mRNA in the brainstem is relatively high, making quantitative assessments of mRNA levels difficult. To combat this problem, we constructed an intron-specific (hnRNA) probe using PCR.

hnRNA probes have been widely used as indices of in vivo transcriptional activity $(40,41)$. hnRNA probes hybridize to intron-specific RNA sequences prior to processing to mature mRNA. The short half-life of hnRNA makes them useful tools to study recent transcriptional activation in individual neurons following specific stimuli $(40,41)$. We found that the relative levels of TH hnRNA were comparatively high in the LC (Figure 6b) even after PFS administration. In addition, a few neurons in the A5, the NTS, the RVLM, and the CVLM displayed a nuclear hybridization signal (Figure $6, \mathrm{~d}, \mathrm{f}, \mathrm{h}$, and $\mathrm{j}$ ). In these regions, neither i.v. nor i.c.v. administration of PFS further induced TH hnRNA expression at any time points examined. In contrast, both i.v. and i.c.v. administration of EXN-4 (i.v., 300 ng; i.c.v., $30 \mathrm{ng}$ ) provoked a robust and rapid increase in $\mathrm{TH}$ hnRNA expression in the A5, the NTS, the RVLM, and the CVLM (Figure 6, c, e, g, and i). Specifically, TH hnRNA expression was induced 15 minutes to 6 hours after i.c.v. injection, and the TH hnRNA expression returned to base line by 24 hours.

\section{Discussion}

Using telemetric methods, we found that central GLP-1R agonists dose-dependently increased MAP and HR in unrestrained, conscious rats. In addition, we found that doses of EXN-4 that increase MAP and HR also induced Fos-IR in several autonomic control sites in the brain

Table 1

Intracerebroventricular EXN-4 activates hypothalamic and brainstem neurons projecting to the spinal cord

$\begin{array}{lcccccc}\begin{array}{l}\text { Region } \\ \text { (atlas level) }\end{array} & \begin{array}{c}\text { FG-IR } \\ \text { (i.c.v. PFS) }\end{array} & \begin{array}{c}\text { FG-IR } \\ \text { (i.c.v. EXN-4) }\end{array} & \begin{array}{c}\text { Double-labeled: } \\ \text { Fos-IR + FG-IR } \\ \text { (i.c.v. PFS) }\end{array} & \begin{array}{c}\text { Double-labeled: } \\ \text { Fos-IR + FG-IR } \\ \text { (i.c.v. EXN-4) }\end{array} & \begin{array}{c}\text { \% Double-labeled: } \\ \text { Fos-IR + FG-IR } \\ / \text { Total FG-IR } \\ \text { (i.c.v. PFS) }\end{array} & \begin{array}{c}\text { \% Double-labeled: } \\ \text { Fos-IR + FG-IR } \\ / \text { Total FG-IR } \\ \text { (i.c.v. EXN-4) }\end{array} \\ \text { PVH (26/26) } & 60 \pm 7.9 & 95 \pm 16.1 & 5 \pm 0.7 & 19 \pm 2.6^{\mathrm{A}} & 8 \pm 1.2 & 21 \pm 1.0^{\mathrm{A}} \\ \text { RCA (27/27) } & 12 \pm 2.2 & 14 \pm 1.1 & 2 \pm 0.0 & 6 \pm 0.3^{\mathrm{A}} & 19 \pm 3.2 & 41 \pm 1.2^{\mathrm{A}} \\ \text { LHA (32/31) } & 86 \pm 3.7 & 108 \pm 19.7 & 5 \pm 0.7 & 13 \pm 1.8^{\mathrm{A}} & 5 \pm 0.7 & 13 \pm 1.1^{\mathrm{A}} \\ \text { LC (60/52) } & 12 \pm 2.2 & 12 \pm 2.6 & 1 \pm 0.3 & 8 \pm 1.2^{\mathrm{A}} & 3.6 & 5 \pm 2.7^{\mathrm{A}} \\ \text { A5 (60/52) } & 13 \pm 1.7 & 14 \pm 2.3 & 1 \pm 0.6 & 4 \pm 0.8^{\mathrm{A}} & 3 \pm 3.6 & 29 \pm 2.4^{\mathrm{A}} \\ \text { RVLM (70/64) } & 35 \pm 2.2 & 18 \pm 0.7 & 1 \pm 0.6 & 9 \pm 0.9^{\mathrm{A}} & 5 \pm 3.5 & 44 \pm 3.4^{\mathrm{A}}\end{array}$

Values represent estimates of mean counts of cells \pm SEM. Data were analyzed by ANOVA and differences between groups by Fischer's PLSD. The atlas level designations correspond to those described by Paxinos and Watson (53) and Swanson (54), respectively. ${ }^{A} P<0.01$ compared with PFS-treated rats; $n=4$ for $\mathrm{PFS}$ group, and $n=4$ for EXN-4-treated rats. 
regions and in the adrenal medulla. In the hypothalamus and the brainstem, we also observed Fos-IR in neurons (including catecholamine neurons) that innervate sympathetic preganglionic neurons. Furthermore, EXN-4 increased TH transcription in medullary catecholamine neurons. These findings suggest that CNS pathways known to be critical regulators of sympathetic outflow are engaged by central GLP-1 neurons to regulate physiological processes including $\mathrm{BP}$ and $\mathrm{HR}$ responses.

GLP-1 neurons are expressed predominantly in the caudal medulla. However, the GLP-1R is widely expressed in the CNS. Available data suggest that central GLP-1 action produces taste aversion (13) and that GLP-1R signaling systems are activated following exposure to agents that serve visceral illness such as lithium chloride $(\mathrm{LiCl})$ or LPS $(12,15)$. These models are internal stressors and induced responses defined as interoceptive stress (16). Interestingly, GLP-1R antagonists attenuate the increase in Fos-IR induced by the administration of LiCl or LPS. Notably, several interoceptive stress models also activate sympathetic outflow $(27,42)$. Taken together, these data suggest that the autonomic alterations characteristic of interoceptive stress may be mediated by central GLP- 1 systems.

Our current findings extend previous reports linking GLP-1 or EXN-4 to increases in MAP and HR in anesthetized rats (18-21) and provide a mechanism invoking catecholaminergic neurons as downstream mediators of GLP-1 action. In anesthetized animals, bilateral vagotomy blocks the stimulatory effects of i.c.v. GLP-1 on BP and HR (20), suggesting that the cardiovascular effects of central GLP-1 systems may be due to mechanisms including inhibition of vagal tone to the cardiovascular system. However, the inherent confounding effects of anesthesia on cardiovascular physiology must be

\footnotetext{
Figure 5

EXN-4 activates medullary catecholamine neurons projecting to the spinal cord. (a-c) Double-label immunohistochemistry demonstrates that neurons that innervate sympathetic preganglionic neurons (brown cytoplasm) also contain i.c.v. EXN-4-induced Fos-IR (black nuclei) in (a) the PVH, (b) the Arc/RCA, and (c) the LHA of the hypothalamus. (d-f) Higher-magnification view of the boxed areas in $\mathbf{a}-\mathbf{c}$, respectively. ( $\mathbf{g}-\mathbf{i})$ Double-label immunohistochemistry demonstrates that $\mathrm{TH}$-immunoreactive neurons (brown cytoplasm) also contain i.c.v. EXN-4-induced Fos-IR (black nuclei) in (g) the NTS, (h) the LC, and (i) the CVLM. (j-I) Higher-magnification view of the boxed areas in $\mathbf{g}-\mathbf{i}$, respectively. ( $\mathbf{m}-\mathbf{p})$ Triple-label immunohistochemistry reveals that many medullary catecholamine neurons (red cytoplasm in $\mathbf{m}$ and $\mathbf{o}$ ) that project to the IML in the spinal cord (green neurons in $\mathbf{n}$ and $\mathbf{p}$ ) also contain i.c.v. EXN-4-induced Fos-IR (black nuclei) in the RVLM ( $\mathbf{m}$ and $\mathbf{n}$ ) and the $A 5$ (o and $\mathbf{p}$ ). Scale bar $=100 \mu \mathrm{m}$ in $\mathbf{a}-\mathbf{c}$ and $\mathbf{g}-\mathbf{i}, 25 \mu \mathrm{m}$ in $\mathbf{d}-\mathbf{f}$ and $\mathbf{j}-\mathbf{I}$, and $50 \mu \mathrm{m}$ in $\mathbf{m}-\mathbf{p}$.
}

considered $(43,44)$. Difference in potential mechanisms of GLP-1 action on the cardiovascular system between various studies may be due in part to experimental differences in the preparation and handling of the animals and the doses of the agonists used in each study. In our system, the threshold dose of i.c.v. EXN-4 to increase MAP is approximately one-tenth to one-hundredth the threshold dose of i.v. EXN-4, and i.c.v. administration of GLP-1R antagonist blocked the effect of i.c.v. EXN-4 on MAP and HR. As GLP- 1 is well known to be a potent stimulator of glucose-dependent insulin secretion, we excluded the possibility that changes in blood glucose following GLP-1 administration might be responsible for induction of Fos activation and counter-regulatory responses leading to sympathetic nervous system activation (3). The demonstration that GLP-1 agonists activate Fos, TH gene transcription, and cardiovascular responses at doses that do not lower blood glucose strongly suggests that central GLP- 1 systems are capable of regulating $\mathrm{BP}$ and HR independent of the established peripheral actions of GLP-1 on glucoregulation.

Based on our findings, we propose that two possible effector pathways downstream of central GLP-1 neurons regulate sympathetic outflow. These two
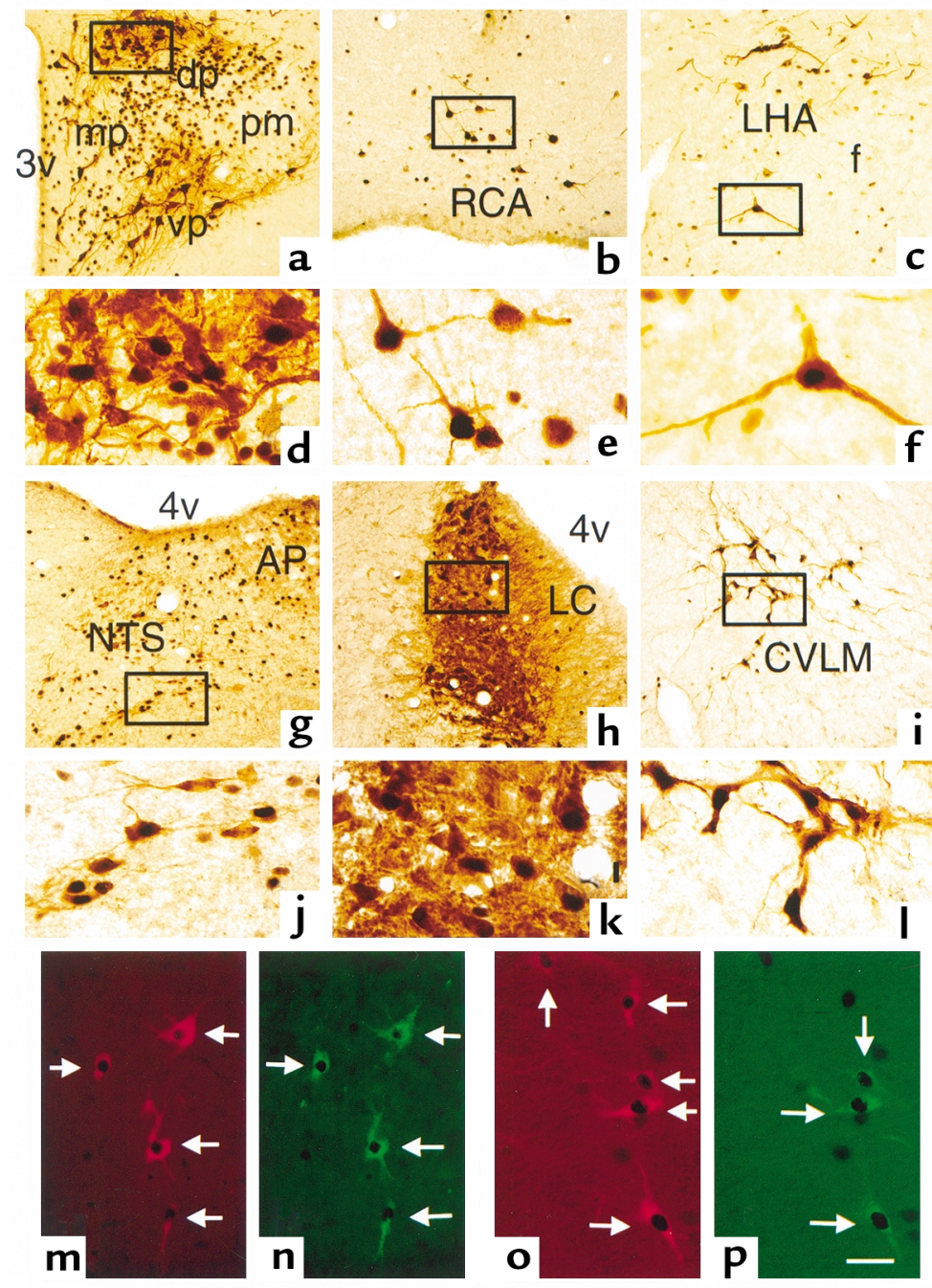

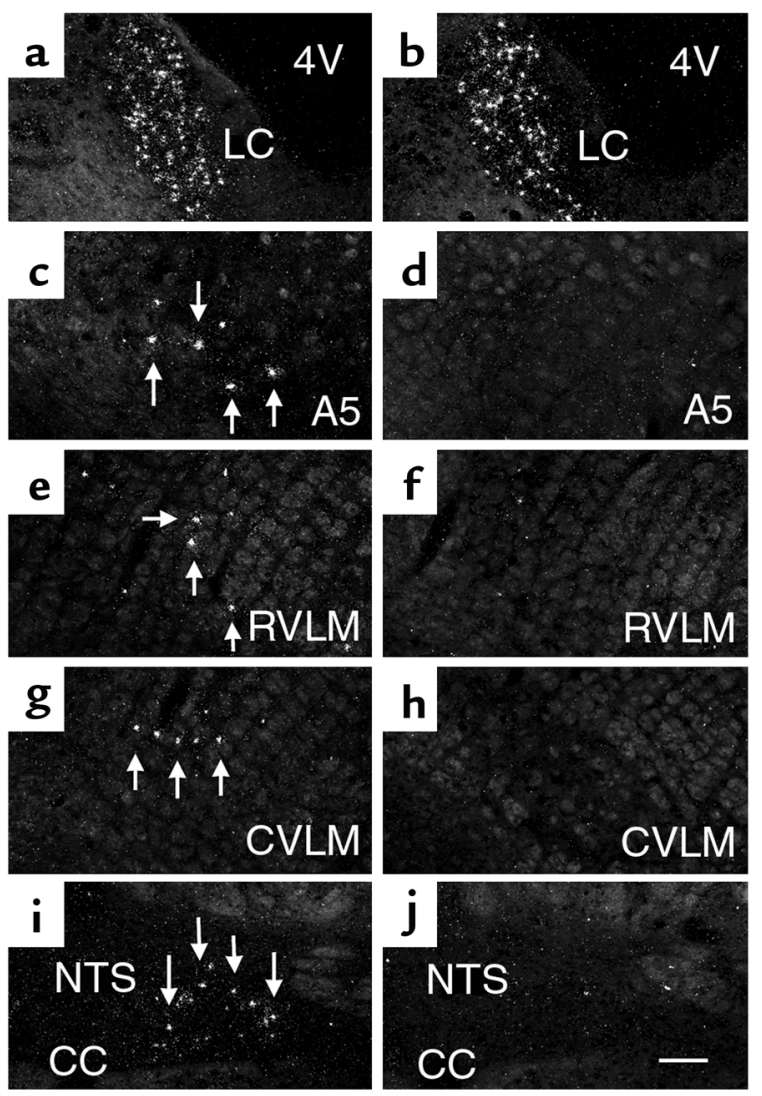

pathways include monosynaptic hypothalamo-spinal and bulbo-spinal projections to sympathetic preganglionic neurons $(32,45)$. Several studies have demonstrated that neurons in the RVLM and A5 provide descending input to sympathetic preganglionic neurons. Additionally, the RVLM provides tonic excitatory input to the cardiovascular system $(34,46-48)$. Therefore, we suggest that central GLP-1 systems are involved in regulating cardiovascular function in part by engaging catecholamine neurons that project to the IML to activate the adrenal medulla. The PVH is composed of functionally distinct subnuclei that regulate endocrine and autonomic responses (31). Within the

\section{Figure 7}

A neuroanatomical model of GLP-1's actions in the rat brain. Circulating GLP-1 engages GLP-1Rs on the hypothalamic neurons in the PVH, Arc, and LHA, and medullary catecholamine neurons in the A5, RVLM, and CVLM, probably through the AP and NTS where GLP-1 neurons are located. These GLP-1-activated neurons in the hypothalamus and brainstem have monosynaptic descending projections to the sympathetic preganglionic neurons in the IML, which innervate the adrenal medulla and heart to increase BP and $H R$ (autonomic responses). This model predicts that some of the medullary catecholamine neurons project to the hypothalamic neurons. GLP-1-activated neurons in the PVH also act on the pituitary to engage neuroendocrine responses.

\section{Figure 6}

Intracerebroventricular EXN-4 activates TH transcription in the medullary catecholamine neurons. All sections were hybridized with a specific TH intronic RNA probe 15 minutes after administration of i.c.v. EXN-4 (left column) or PFS (right column) in several brainstem regions, including ( $\mathbf{a}$ and $\mathbf{b})$ the LC, (c and $\mathbf{d})$ the A5, (e and $\mathbf{f})$ the RVLM, ( $\mathbf{g}$ and $\mathbf{h}$ ) the CVLM, and ( $\mathbf{i}$ and $\mathbf{j}$ ) the caudal part of the NTS. CC, central canal. Scale bar $=100 \mu \mathrm{m}$.

PVH, EXN-4 induced Fos-IR in the medial parvicellular neurons and in the ventral, dorsal, and lateral subdivisions, suggesting that central GLP-1 systems may regulate the hypothalamo-pituitary-adrenocortical (HPA) axis and autonomic outflow. Indeed, neurons in the caudal NTS that project to the PVH contain GLP-1 (17). GLP-1 also activates the HPA axis, presumably through stimulation of CRH neurons (17). With respect to autonomic responses, the transneuronal tracing has identified the $\mathrm{PVH}$ as one of a few brain sites that regulate the entire sympathetic outflow (49). Our current study suggests that GLP-1 acts in the PVH to mediate neuroendocrine responses and to modulate sympathetic outflow.

In our current study, both peripheral and central administration of GLP-1 agonists rapidly increased $\mathrm{BP}$ and HR and induced similar patterns of Fos-IR throughout the brain, including several key autonomic regulatory sites. The induction of Fos-IR in these sites is consistent with the concept that the increased activity of these neurons contributes to the increases in BP and HR. However, we cannot rule out the possibility that the expression of Fos in some of these sites may simply be secondary to the increased $\mathrm{BP}$ and HR and may not represent activation of neurons inducing the observed responses. Still, the Fos patterns induced by GLP-1 agonists in the brainstem are distinct from patterns induced by phenylephrineinduced hypertension, which does not induce Fos-IR in catecholamine neurons (50). Indeed, the patterns of GLP-1-induced Fos are more similar to those of stimuli that increase HR and BP, increase Fos-IR in TH neurons, and activate TH mRNA $(36,50)$. Thus,

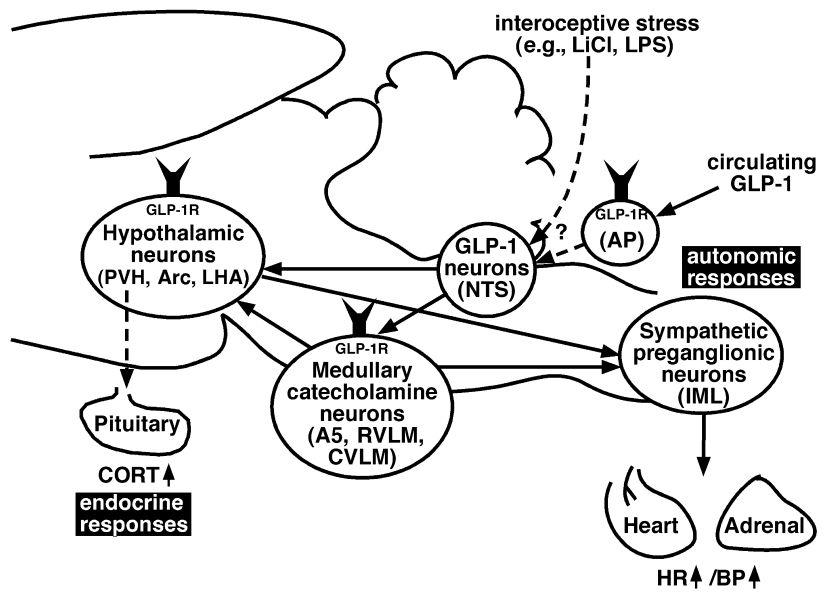


we suggest that Fos induction in central autonomic control sites by GLP-1 agonists is not likely to be a secondary consequence of increased BP and HR.

One interesting difference in the patterns of i.v. and i.c.v. injections was that in the AP. Specifically, i.v. EXN-4 induced much more Fos-IR than did i.c.v. EXN-4 in the AP. GLP-1Rs are prominently expressed in the AP, a circumventricular organ that lacks a blood-brain barrier. Therefore, it is likely that neurons in the AP have access to circulating GLP-1. Indeed, recent studies using ${ }^{125}$ I-GLP-1 have demonstrated that GLP-1Rs are accessible to peripheral GLP- 1 in the subfornical organ and the AP in the rat (51). Furthermore, in our preliminary study, we found that i.v. EXN-4 induced Fos-IR in GLP-1 neurons, suggesting that peripheral EXN-4 activates the central GLP-1 system, which may contribute to the effects we observed. Consistent with this hypothesis, Barragan et al. (20) showed that i.c.v. administration of a GLP-1 antagonist blocked cardiovascular responses to systemically injected GLP- 1 . Therefore, it is plausible that circulating GLP-1 activates GLP1 -responsive neurons in the $\mathrm{AP}$ and also activates GLP-1 neurons in the underlying NTS. This model would predict that GLP-1 neurons in turn activate neurons expressing GLP-1Rs in the hypothalamus and the brainstem, leading to coordinated endocrine and autonomic effects (Figure 7).

The data reported here suggest that the central GLP-1 system can regulate sympathetic outflow. In normal humans and rodents, circulating levels of GLP-1 are low in the fasting state, rise following nutrient ingestion, and fall within minutes due to rapid enzymatic inactivation by DPP-IV. Indeed, continuous infusion of GLP-1 does not increase BP and HR in human subjects with type 2 diabetes (52). In contrast, more potent degradation-resistant long-acting GLP-1 analogues, including EXN-4, are being considered as therapeutic agents for the treatment of type 2 diabetes. Our current results demonstrate a dosedependent increase in BP and HR following both central and peripheral administration of EXN-4. However, the pathophysiological significance of our findings in rodents must be assessed in further studies that carefully examine dose response relationships in both acute and chronic paradigms, especially in diabetic subjects. Nonetheless, our results suggest that the central GLP-1 system is a regulator of sympathetic outflow and raise questions about the potential consequences of sustained GLP-1R activation for the treatment of patients with type 2 diabetes.

\section{Acknowledgments}

The authors thank Quan Ha, Toshiro Kishi, Im Jihee, Brian J. Choi, Hiromichi Kashihara, Takuma Kishimoto, and Hiroshi Muraki for technical assistance. This work was supported by NIH grants DK-59751 and DK-56116 and Juvenile Diabetes Research Foundation grant 2000-559.
1. Creutzfeldt, W. 2001. The entero-insular axis in type 2 diabetes: incretins as therapeutic agents. Exp. Clin. Endocrinol. Diabetes. 109:S288-S303.

2. Goke, R., et al. 1993. Exendin-4 is a high potency agonist and truncated exendin-(9-39)-amide an antagonist at the glucagon-like peptide 1-(7-36)-amide receptor of insulin-secreting beta-cells. J. Biol. Chem. 268:19650-19655.

3. Young, A.A., et al. 1999. Glucose-lowering and insulin-sensitizing actions of exendin-4: studies in obese diabetic $(o b / o b, d b / d b)$ mice, diabetic fatty Zucker rats, and diabetic rhesus monkeys (Macaca mulatta). Diabetes. 48:1026-1034.

4. Drucker, D.J. 1998. Glucagon-like peptides. Diabetes. 47:159-169.

5. Egan, J.M., Clocquet, A.R., and Elahi, D. 2002. The insulinotropic effect of acute exendin- 4 administered to humans: comparison of nondiabetic state to type 2 diabetes. J. Clin. Endocrinol. Metab. 87:1282-1290.

6. Jin, S.L., et al. 1988. Distribution of glucagonlike peptide I (GLP-I), glucagon, and glicentin in the rat brain: an immunocytochemical study. J. Comp. Neurol. 271:519-532.

7. Larsen, P.J., Tang-Christensen, M., Holst, J.J., and Orskov, C. 1997. Distribution of glucagon-like peptide- 1 and other preproglucagon-derived peptides in the rat hypothalamus and brainstem. Neuroscience. 77:257-270.

8. Merchenthaler, I., Lane, M., and Shughrue, P. 1999. Distribution of prepro-glucagon and glucagon-like peptide- 1 receptor messenger RNAs in the rat central nervous system. J. Comp. Neurol. 403:261-280.

9. Turton, M.D., et al. 1996. A role for glucagon-like peptide- 1 in the central regulation of feeding. Nature. 379:69-72.

10. Van Dijk, G., et al. 1996. Central infusions of leptin and GLP-1-(7-36) amide differentially stimulate c-FLI in the rat brain. Am. J. Physiol. 271:R1096-R1100.

11. Edwards, C.M., et al. 2001. Exendin-4 reduces fasting and postprandial glucose and decreases energy intake in healthy volunteers. Am. J. Physiol. Endocrinol. Metab. 281:E155-E161.

12. Thiele, T.E., et al. 1997. Central infusion of GLP-1, but not leptin, produces conditioned taste aversions in rats. Am. J. Physiol. 272:R726-R730.

13. Seeley, R.J., et al. 2000. The role of CNS glucagon-like peptide-1 (7-36) amide receptors in mediating the visceral illness effects of lithium chloride. J. Neurosci. 20:1616-1621.

14. Rinaman, L. 1999. Interoceptive stress activates glucagon-like peptide1 neurons that project to the hypothalamus. Am. J. Physiol. 277:R582-R590.

15. Rinaman, L. 1999. A functional role for central glucagon-like peptide1 receptors in lithium chloride-induced anorexia. Am. J. Physiol. 277:R1537-R1540.

16. Sawchenko, P.E., Li, H.Y., and Ericsson, A. 2000. Circuits and mechanisms governing hypothalamic responses to stress: a tale of two paradigms. Prog. Brain Res. 122:61-78.

17. Larsen, P.J., Tang-Christensen, M., and Jessop, D.S. 1997. Central administration of glucagon-like peptide-1 activates hypothalamic neuroendocrine neurons in the rat. Endocrinology. 138:4445-4455.

18. Barragan, J.M., Rodriguez, R.E., and Blazquez, E. 1994. Changes in arterial blood pressure and heart rate induced by glucagon-like peptide-1(7-36) amide in rats. Am. J. Physiol. 266:E459-E466.

19. Barragan, J.M., Rodriguez, R.E., Eng, J., and Blazquez, E. 1996. Interactions of exendin-(9-39) with the effects of glucagon-like peptide-1-(736) amide and of exendin- 4 on arterial blood pressure and heart rate in rats. Regul. Pept. 67:63-68.

20. Barragan, J.M., Eng, J., Rodriguez, R., and Blazquez, E. 1999. Neural contribution to the effect of glucagon-like peptide-1-(7-36) amide on arterial blood pressure in rats. Am. J. Physiol. 277:E784-E791.

21. Bojanowska, E., and Stempniak, B. 2000. Effects of centrally or systemically injected glucagon-like peptide-1 (7-36) amide on release of neurohypophysial hormones and blood pressure in the rat. Regul. Pept. 91:75-81.

22. Williams, T.D., et al. 2000. Concurrent reductions in blood pressure and metabolic rate during fasting in the unrestrained SHR. Am. J. Physiol. Regul. Integr. Comp. Physiol. 278:R255-R262.

23. Elmquist, J.K., Scammell, T.E., Jacobson, C.D., and Saper, C.B. 1996. Distribution of Fos-like immunoreactivity in the rat brain following intravenous lipopolysaccharide administration. J. Comp. Neurol. 371:85-103.

24. Scammell, T.E., Elmquist, J.K., Griffin, J.D., and Saper, C.B. 1996. Ventromedial preoptic prostaglandin E2 activates fever-producing autonomic pathways. J. Neurosci. 16:6246-6254.

25. Cechetto, D.F., and Saper, C.B. 1988. Neurochemical organization of the hypothalamic projection to the spinal cord in the rat. J. Comp. Neurol. 272:579-604.

26. Elias, C.F., et al. 1998. Leptin activates hypothalamic CART neurons projecting to the spinal cord. Neuron. 21:1375-1385.

27. Zhang, Y.H., Lu, J., Elmquist, J.K., and Saper, C.B. 2000. Lipopolysaccharide activates specific populations of hypothalamic and brainstem neurons that project to the spinal cord. J. Neurosci. 20:6578-6586. 
28. Brown, E.R., Coker, G.T., III, and K.L. O’Malley. 1987. Organization and evolution of the rat tyrosine hydroxylase gene. Biochemistry. 26:5208-5212

29. Marcus, J.N., et al. 2001. Differential expression of orexin receptors 1 and 2 in the rat brain. J. Comp. Neurol. 435:6-25.

30. Ericsson, A., Liu, C., Hart, R.P., and Sawchenko, P.E. 1995. Type 1 interleukin-1 receptor in the rat brain: distribution, regulation, and relationship to sites of IL-1-induced cellular activation. J. Comp. Neurol. 361:681-698.

31. Saper, C.B. 1995. Central autonomic system. Academic Press Inc. San Diego, California, USA. 107-135.

32. Saper, C.B., Loewy, A.D., Swanson, L.W., and Cowan, W.M. 1976. Direct hypothalamo-autonomic connections. Brain Res. 117:305-312.

33. Sawchenko, P.E., and Swanson, L.W. 1982. Immunohistochemical identification of neurons in the paraventricular nucleus of the hypothalamus that project to the medulla or to the spinal cord in the rat. J. Comp. Neurol. 205:260-272.

34. Guyenet, P.G., Haselton, J.R., and Sun, M.K. 1989. Sympathoexcitatory neurons of the rostroventrolateral medulla and the origin of the sympathetic vasomotor tone. Prog. Brain Res. 81:105-116.

35. Reis, D.J., Ruggiero, D.A., and Morrison, S.F. 1989. The C1 area of the rostral ventrolateral medulla oblongata. A critical brainstem region for control of resting and reflex integration of arterial pressure. Am. J. Hypertens. 2:363S-374S.

36. Chan, R.K., and Sawchenko, P.E. 1995. Hemodynamic regulation of tyrosine hydroxylase messenger RNA in medullary catecholamine neurons: a c-fos-guided hybridization histochemical study. Neuroscience. 66:377-390.

37. Ritter, S., Bugarith, K., and Dinh, T.T. 2001. Immunotoxic destruction of distinct catecholamine subgroups produces selective impairment of glucoregulatory responses and neuronal activation. J. Comp. Neurol. 432:197-216.

38. Zigmond, E.R. 1985. Biochemical consequences of synaptic stimulation: the regulation of tyrosine hydroxylase activity by multiple transmitters. Trends Neurosci. 8:63-69.

39. Biguet, N.F., Rittenhouse, A.R., Mallet, J., and Zigmond, R.E. 1989. Preganglionic nerve stimulation increases mRNA levels for tyrosine hydroxylase in the rat superior cervical ganglion. Neurosci. Lett. 104:189-194.

40. Herman, J.P., Schafer, M.K., Watson, S.J., and Sherman, T.G. 1991. In situ hybridization analysis of arginine vasopressin gene transcription using intron-specific probes. Mol. Endocrinol. 5:1447-1456.

41. Kovacs, K.J., and Sawchenko, P.E. 1996. Sequence of stress-induced alterations in indices of synaptic and transcriptional activation in par- vocellular neurosecretory neurons. J. Neurosci. 16:262-273.

42. Ericsson, A., Kovacs, K.J., and Sawchenko, P.E. 1994. A functional anatomical analysis of central pathways subserving the effects of interleukin-1 on stress-related neuroendocrine neurons. J. Neurosci. 14:897-913.

43. Stornetta, R.L., Guyenet, P.G., and McCarty, R.C. 1987. Autonomic nervous system control of heart rate during baroreceptor activation in conscious and anesthetized rats. J. Auton. Nerv. Syst. 20:121-127.

44. Bachelard, H., Gardiner, S.M., and Bennett, T. 1990. Cardiovascular responses elicited by chemical stimulation of the rostral ventrolateral medulla in conscious, unrestrained rats. J. Auton. Nerv. Syst. 31:185-190.

45. Loewy, A.D., McKellar, S., and Saper, C.B. 1979. Direct projections from the A5 catecholamine cell group to the intermediolateral cell column. Brain Res. 174:309-314.

46. Ross, C.A., Ruggiero, D.A, Joh, T.H., Park, D.H., and Reis, D.J. 1984. Rostral ventrolateral medulla: selective projections to the thoracic autonomic cell column from the region containing $\mathrm{C} 1$ adrenaline neurons. J. Comp. Neurol. 228:168-185.

47. Loewy, A.D., Marson, L., Parkinson, D., Perry, M.A., and Sawyer, W.B. 1986. Descending noradrenergic pathways involved in the A5 depressor response. Brain Res. 386:313-324.

48. Huangfu, D.H., Koshiya, N., and Guyenet, P.G. 1991. A5 noradrenergic unit activity and sympathetic nerve discharge in rats. Am. J. Physiol. 261:R393-R402.

49. Strack, A.M., Sawyer, W.B., Hughes, J.H., Platt, K.B., and Loewy, A.D. 1989. A general pattern of CNS innervation of the sympathetic outflow demonstrated by transneuronal pseudorabies viral infections. Brain Res. 491:156-162.

50. Chan, R.K., and Sawchenko, P.E. 1994. Spatially and temporally differentiated patterns of $\mathrm{c}$-fos expression in brainstem catecholaminergic cell groups induced by cardiovascular challenges in the rat. J. Comp. Neu rol. 348:433-460.

51. Orskov, C., Poulsen, S.S., Moller, M., and Holst, J.J. 1996. Glucagon-like peptide I receptors in the subfornical organ and the area postrema are accessible to circulating glucagon-like peptide I. Diabetes. 45:832-835.

52. Toft-Nielsen, M.B., Madsbad, S., and Holst, J.J. 1999. Continuous subcutaneous infusion of glucagon-like peptide 1 lowers plasma glucose and reduces appetite in type 2 diabetic patients. Diabetes Care. 22:1137-1143.

53. Paxinos, G., and Watson, C. 1997. The rat brain, in stereotaxic coordinates. Compact 3rd edition. Academic Press Inc. San Diego, California, USA $78 \mathrm{pp}$.

54. Swanson, L.W. 1992 Brain maps: structure of the rat brain. Volume 1. Elsevier. Amsterdam, The Netherlands. 240 pp. 